\title{
Optimization and activation of renewable durian husk for biosorption of lead (II) from a aqueous medium
}

\begin{abstract}
Background: Threonine is an essential amino acid that is extensively used in livestock industry as feed supplement due to its pronounced effect in improving the growth performance of animals. Application of genetically engineered bacteria for amino acid production has its share of controversies after eosinophils myalgia syndrome outbreak in 1980s. This has urged for continuous search for a food grade producer as a safer alternative for industrial amino acid production. Lactic acid bacteria (LAB) appear as an exceptional candidate owing to their nonpathogenic nature and reputation of Generally Recognized as Safe (GRAS) status. Recently, we have identified a LAB, Pediococcus pentosaceus TL-3, isolated from Malaysian food as a potential threonine producer. Thus, the objective of this study was to enhance the threonine production by $\mathrm{P}$. pentosaceus TL-3 via optimized medium developed by using PlackettBurman design (PBD) and central composite design (CCD). Results: Molasses, meat extract, (NH4)2SO4, and MnSO4 were identified as the main medium components for threonine production by P. pentosaceus TL-3. The optimum concentration of molasses, meat extract, (NH4)2SO4 and MnSO4 were found to be $30.79 \mathrm{~g} / \mathrm{L}, 25.30 \mathrm{~g} / \mathrm{L}, 8.59 \mathrm{~g} / \mathrm{L}$, and $0.098 \mathrm{~g} / \mathrm{L}$ respectively based on model obtained in $\mathrm{CCD}$ with a predicted net threonine production of $123.07 \mathrm{mg} / \mathrm{L}$. The net threonine production by P. pentosaceus TL-3 in the optimized medium was enhanced approximately 2 folds compared to the control. Conclusions: This study has revealed the potential of P. pentosaceus TL-3 as a safer alternative to produce threonine. Additionally, the current study has identified the key medium components affecting the production of threonine by P. pentosaceus TL-3, followed by optimization of their concentrations by means of statistical approach. The findings of this study could act as a guideline for the future exploration of amino acid production by LAB.
\end{abstract}

Keyword: Lead (II) biosorption; Durian husk; Activated carbon; Optimization; Wastewater 